\title{
Prevalidation of an ELISA for Detection of a New Clinical Entity: Leishmania donovani-Induced Cutaneous Leishmaniasis
}

\author{
Bhagya Deepachandi, ${ }^{1}$ Sudath Weerasinghe, ${ }^{1}$ Himali Gunathilake, ${ }^{1}$ \\ Thisira P. Andrahennadi, ${ }^{2}$ Mahendra N. Wickramanayake, ${ }^{3}$ Shantha Siri, ${ }^{4}$ \\ Vishvanath Chandrasekharan, ${ }^{3}$ Preethi Soysa, ${ }^{2}$ and Yamuna Siriwardana ${ }^{1}{ }^{1}$ \\ ${ }^{1}$ Department of Parasitology, Faculty of Medicine, University of Colombo, Colombo 00800, Sri Lanka \\ ${ }^{2}$ Department of Biochemistry and Molecular Biology, Faculty of Medicine, University of Colombo, Colombo 00800, Sri Lanka \\ ${ }^{3}$ Department of Chemistry, Faculty of Science, University of Colombo, Colombo 00300, Sri Lanka \\ ${ }^{4}$ National Science Foundation, 47/5 Maitland Place, Colombo 00700, Sri Lanka
}

Correspondence should be addressed to Yamuna Siriwardana; yamuna@parasit.cmb.ac.lk

Received 15 March 2020; Accepted 29 June 2020; Published 15 July 2020

Academic Editor: Danilo Corradini

Copyright (c) 2020 Bhagya Deepachandi et al. This is an open access article distributed under the Creative Commons Attribution License, which permits unrestricted use, distribution, and reproduction in any medium, provided the original work is properly cited.

\begin{abstract}
Human leishmaniasis which is considered a neglected tropical parasitic disease presents in three main clinical forms (i.e., cutaneous leishmaniasis (CL), mucocutaneous leishmaniasis (MCL), and visceral leishmaniasis (VL)) that are mainly determined by its causative species. Leishmania donovani, the most virulent and visceralizing parasite, is increasingly reported to cause CL in many countries in the world. Although CL is generally not considered to evoke a humoral immune response except for a nonrobust and a variable response in minority of cases, VL is associated with a clear strong humoral response. However, humoral response in $L$. donovani-induced CL has not been well evaluated before. A suitable serology-based assay is an essential primary step in such a study. An indirect enzyme-linked immunosorbent assay (ELISA) based on Leishmania promastigote crude antigen $(\mathrm{Ag})$ was designed and optimized in order to utilize in further serological studies on this new clinical entity. Optimization included quantification of crude Ag, checkerboard titration method for determination of optimal concentrations for coating Ag, human sera and secondary antibody $(\mathrm{Ab})$ with suitable coating buffer, blocking buffer, and incubating temperatures. The selected coating buffer was $0.02 \mathrm{M}$ phosphate buffer, $\mathrm{pH} 6.8$, and the blocking buffer was $2 \%$ fetal bovine serum with $0.01 \mathrm{M}$ phosphate-buffered saline. At least $1 \mu \mathrm{g}$ of crude Ag was required for coating the ELISA plate, while 1:1000 serum was used as primary Ab. The optimized concentration of secondary Ab was $1: 64000$ which might be altered according to manufacturer recommendations. The assay specificity was pre-evaluated using sera ( $n=20$ from each category) from confirmed CL patients and controls (other skin diseases which mimic CL, other systemic diseases that mimic VL, nonendemic healthy controls, and endemic healthy controls). This procedure described an optimization procedure of an ELISA technique for detection of anti-Leishmania antibodies in patients with $L$. donovani caused CL.
\end{abstract}

\section{Introduction}

Leishmaniasis, a parasitic disease found in parts of the tropics, subtropics, and Southern Europe present as one of the three clinical forms: cutaneous leishmaniasis (CL), visceral leishmaniasis (VL), and mucocutaneous leishmaniasis (MCL) which are mainly species dependant. Untreated VL and MCL potentially fatal while CL is associated with great morbidity [1]. CL induced by $L$. donovani which is the known cause of VL is increasingly reported at a global scale [2-4]. Humoral response against the mismatching clinical and parasite scenario has not been examined so far [5].

Sri Lanka sets an ideal example in the southasian region where more than 6000 cases of CL have been notified during past 10 years [6]. L. donovani was identified as the causative agent of CL in this setting [7-10]. Main clinical entity remained as $\mathrm{CL}$ in a clear majority of reported cases over the time [11-16]. 
Skin and mucosal infections result mainly in cell-mediated immunity, while visceralizing parasites exert a humoral response [17]. This has enabled development and effective utilization of many serological tools for diagnosis of VL [18-22]. Usefulness of serology in diagnosis of CL has shown to be less useful with highly variable and usually weak response [23, 24].

However, owing to the unexamined visceralizing potential of the local causative species, examination of the serological response was thought to be useful in this clinical entity. Further confirming this, two preliminary studies reported a seroprevalence among local CL during the first attempt $[5,25]$. The seroprevalence was initially found as $14.0 \%$ [25]. Subsequently, a seroprevalence of $34.0 \%$ was reported with modifications to the technique indicating the possibility for further improvement [5]. However, specificity of the tool was not examined during these two attempts. Meanwhile, changing trends in CL profile with different disease transmission foci, atypical CL forms, and emergence of VL and MCL have also been reported in the country, justifying the further examination of serological aspects of $L$. donovani-induced CL $[16,26-30]$.

Accurate determination of the actual seroprevalence also enables decision-making with regard to the usefulness of its further development as a diagnostic or a research tool. The aim of this study was to further optimize and examine the specificity of the previously established ELISA technique with a view to revisit the seroprevalence of $L$. donovaniinduced CL.

\section{Materials and Methods}

2.1. Setting and Timing. This study was carried out in Sri Lanka on locally acquired cases of CL reported in local patients diagnosed during 2014-2016.

2.2. Instrumentation, Materials, and Reagents. Absorbance measurements were obtained by the Thermo Electron Corporation Multiskan EX microplate reader. Micropipettes (0-20 $\mu \mathrm{l}, 20-200 \mu \mathrm{l}$, and 100-1000 $\mu \mathrm{l}$ Nichipet EXII micropipettes from Nichiryo) and microwell plates (96 wells) (Sterilin, Tentorio, Italy) were used. Horseradish peroxidase conjugate-goat anti-human immunoglobulin (gamma chain) secondary Ab (goat anti-human IgG-HRP) and 3,3',5,5-tetramethylbenzidine (TMB) chromogen substrate solution were purchased from Invitrogen (Camarillo, California). Penicillin-streptomycin (Penstrep), heat-inactivated fetal bovine serum (HI-FBS) and medium 199, Hank's balanced salts (M199), and the reagents required for cell culturing were purchased from Gibco (Life Technologies, Grand Island, United States of America). All other chemicals and reagents including, sodium phosphate dibasic $\left(\mathrm{Na}_{2} \mathrm{HPO}_{4}\right)$, sodium phosphate monobasic $\left(\mathrm{NaH}_{2} \mathrm{PO}_{4}\right)$, sodium chloride $(\mathrm{NaCl})$, sodium bicarbonate $\left(\mathrm{NaHCO}_{3}\right)$ potassium chloride $(\mathrm{KCl})$, potassium phosphate monobasic $\left(\mathrm{KH}_{2} \mathrm{PO}_{4}\right)$, sodium carbonate $\left(\mathrm{Na}_{2} \mathrm{CO}_{3}\right)$, copper sulfate $\left(\mathrm{CuSO}_{4}\right)$, potassium sodium tartrate $\left(\mathrm{KNaC}_{4} \mathrm{H}_{4} \mathrm{O}_{6}\right)$, sodium hydroxide $(\mathrm{NaOH})$, Folin and Ciocalteu's phenol reagent, bovine serum albumin (BSA/fraction V), polyethylene glycol sorbitan monolaurate (Tween-20), and sulfuric acid $\left(\mathrm{H}_{2} \mathrm{SO}_{4}\right)$ were purchased from Sigma-Aldrich (now known as Merck, Saint Louis, Missouri, USA).

2.3. Patient Isolates and Preparation of Crude Ag. Clinically suspected patients $(n=35)$ with locally acquired CL were recruited after informed written consent [14]. Lesion aspirates or slit skin scrapings were collected. Diagnosis of CL was confirmed/excluded using light microscopy (LM), in vitro culturing (IVC), and/or polymerase chain reaction (PCR) [31-33].

Clinical isolates were used to inoculate complete medium 199 (M199) supplemented with 20\% HI-FBS and 0.1\% Penstrep [34]. Parasites at the late log phase with an average density of $1 \times 10^{7}$ cells $/ \mathrm{ml}$ were harvested [34]. Crude Ag was extracted from the harvested promastigotes of Leishmania using the freeze-thawing method [35]. The pellet was washed four times in cold $0.01 \mathrm{M}$ phosphate-buffered saline (PBS), $\mathrm{pH} 7.4$, and resuspended at a concentration of $1.0 \mathrm{~g}$ of cell pellet in $2 \mathrm{ml}$ of cold $0.01 \mathrm{M}$ PBS, pH 7.4. Subsequently, the suspension was freeze-thawed (freezing for 30 seconds in liquid nitrogen and thawing at room temperature) for three times. The suspension contained the total crude Ag, and it was aliquoted and stored at $-20^{\circ} \mathrm{C}$.

2.4. Protein Estimation. Extracted crude Ag was quantified using a modified Lowry assay which was developed and validated within home laboratory settings [36]. Briefly, BSA was used as the standards $(100-500 \mu \mathrm{g} / \mu \mathrm{l})$. Standards or the crude Ag sample $(100 \mu \mathrm{l})$ was added to separate wells and mixed with $20 \mu \mathrm{l}$ of $\mathrm{NaOH}(2 \mathrm{~N})$ in a plate shaker for 10 minutes. A volume of $100 \mu \mathrm{l}$ of reagent mixture A (2\% $\mathrm{Na}_{2} \mathrm{CO}_{3}, 1 \% \mathrm{CuSO}_{4}$ and $2 \% \mathrm{KNaC}_{4} \mathrm{H}_{4} \mathrm{O}_{6}$ in $100: 1: 1$ ratio) was added to each well and mixed well for 5 minutes followed by incubation at room temperature for 10 minutes. Folin and Ciocalteu's phenol reagent $(2 \mathrm{~N}, 20 \mu \mathrm{l})$ was added and mixed well immediately and incubated at room temperature in dark conditions for 30 minutes. Absorbance was read at $650 \mathrm{~nm}$ using an ELISA reader.

2.5. Indirect ELISA. A ninety-six-well ELISA plate (Sterilin, Tentorio, Italia) was coated with $100 \mu$ l of extracted crude protein (containing at least $1 \mu \mathrm{g}$ protein) dissolved in $0.02 \mathrm{M}$ phosphate buffer, $\mathrm{pH} 6.8$, and incubated over night at $4{ }^{\circ} \mathrm{C}$ $[21,22]$. Thereafter, the coated wells were washed 3 times with $0.01 \mathrm{M}$ phosphate-buffered saline (PBS) with $0.1 \%$ tween-20 (PBST) and incubated with $200 \mu \mathrm{l}$ of blocking buffer (0.01 M PBS with 2\% FBS) at room temperature for 6 to 8 hours. Reference Abs (sera) at appropriate dilutions were added and incubated overnight at $4^{\circ} \mathrm{C}[21,22]$. Following overnight incubation, the plate was washed with PBST for 3 times and $100 \mu \mathrm{l}$ goat anti-human IgG-HRP (at appropriate dilution) was added to each well. After 30 minutes at $37^{\circ} \mathrm{C}$, the plate was washed with PBST for 6 times with 5 minutes gentle shaking for last 5 washings. Subsequently, the plate was incubated with $100 \mu \mathrm{l}$ of TMB 
(tetramethyl benzidine) substrate solution at room temperature for 30 minutes, and substrate reaction was stopped by adding $100 \mu \mathrm{l}$ of $1 \mathrm{~N} \mathrm{H}_{2} \mathrm{SO}_{4}$. Absorbance values were read at $450 \mathrm{~nm}$ using an ELISA reader [21, 22].

2.6. Checkerboard Titration (CBT) of ELISA. Concentration of reagents required for indirect ELISA (Figure 1) including crude Ag, human serum (primary Ab), and goat anti-human IgG-HRP (secondary $\mathrm{Ab}$ ) was determined using the CBT method described by Crowther [37]. Initially, crude Ag was diluted rowwise on the ELISA plate with known amounts of protein [37]. Preliminary studies were carried out (data are not shown) to determine the best range of $\mathrm{Ag}(0.5-2.5 \mu \mathrm{g})$ for coating the ELISA plate. Only diluent (PBS) was added to the last row. Sera were diluted columnwise (preliminary studies were carried out to determine the best range of serum dilution (1:500-1:5000); data are not shown), and only diluent (PBS-FBS) was added to the last column [37]. Antispecies conjugate was added at single dilution (1:4000 is the recommended dilution from the commercial supplier). The best Ag dilution was estimated where there was an adequate color intensity as a result of binding with antibodies [37].

Using the optimized amount of coating Ag, the optimized dilutions of primary and secondary $A b$ were determined. The plate was coated with optimized amount of coating Ag, and the dilution range of the serum was added as described. The serum was diluted columnwise on the ELISA plate starting with the highest concentration, while antispecies conjugate was diluted rowwise on the ELISA plate (preliminary studies were carried out to determine the best dilution range of anti-species conjugate $(1: 32000-1: 64000)$; data are not shown). The best dilutions at the point of a high binding ratio (BR) with a high assay sensitivity were selected as the optimized dilutions of two reagents.

2.7. Serum Collection, Preparation, and Specificity Assay. Serum samples from confirmed locally acquired CL $(n=20)$ cases and equal numbers $(n=20)$ from each group of controls including NCL, NVL, NEHC, and EHC were used for the study. If positive patients for CL were with a history of overseas travel within two years prior to diagnosis, they were excluded from the study. NCL samples were collected from patients admitted to Dermatology Ward at National Hospital of Sri Lanka (NHSL) which included patients with infectious skin conditions (leprosy and cutaneous tuberculosis $(n=8)$ ) and immunological conditions (contact dermatitis, eczema, and psoriasis $(n=12)$ ). NVL samples were collected from patients with other systemic infectious diseases (dengue, leptospirosis, pyrexia of unknown origin, and hepatomegaly with/or splenomegaly $(n=13))$ and immunological disorder (carcinoma and systemic lupus erythematosus $(n=7))$. Sera were collected from the healthy persons who lived in the western province which is not considered as a leishmanial area $(n=20)$ and healthy persons who lived in Southern Province, a disease-endemic area $(n=20)$.

Laboratory confirmation of samples was done by LM, IVC, and/or PCR [31-33]. Venous blood (approximately

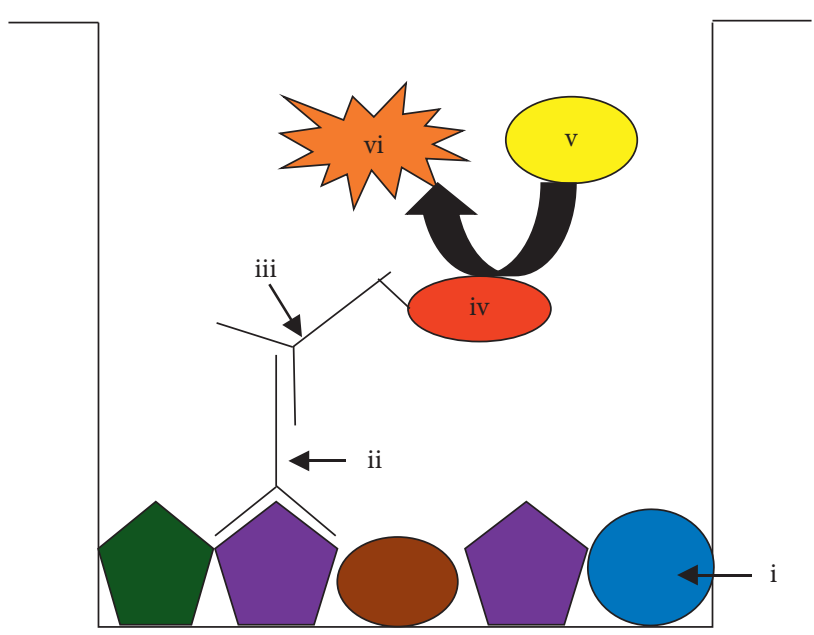

FIGURE 1: Schematic representation of indirect ELISA method used for the study. (i) Crude Leishmania Ag, (ii) anti-Leishmania IgG Ab in human serum, (iii) peroxidase-goat anti-human IgG secondary $\mathrm{Ab}$, (iv) HRP, (v) TMB substrate, and (vi) oxidized TMB.

about $3 \mathrm{cc}$ ) was collected from each participant to plain blood tubes. They were incubated at room temperature for 30 minutes to 1 hour to allow for blood clotting. Sera were separated by centrifugation at $2500 \mathrm{rpm}$ for 10-15 minutes, aliquoted, and stored at $-20^{\circ} \mathrm{C}$ for later use.

Optimized conditions were applied for pre-evaluation of the assay for specific detection of leishmaniasis. Unless otherwise stated, a minimum of two replicates were used for determining mean optical density values of samples in quantification of ELISA absorbance values.

\section{Results}

According to the modified Lowry assay method, the yield of the crude Ag extract was $15 \mathrm{mg}$ for $1 \mathrm{~g}$ of the parasite cell pellet [36]. Selected amounts of crude $\mathrm{Ag}(0.5 \mu \mathrm{g}, 1.5 \mu \mathrm{g}$, and $2.5 \mu \mathrm{g}$ ) were initially used for coating the microwell plate, and the outcome of ELISA was checked. Five dilutions of primary Ab (1:500, $1: 1000,1: 2000,1: 4000$, and $1: 5000)$ were used (Figure 2). Results obtained for a positive serum was compared with the results obtained for a healthy control, and BRs were calculated. A higher BR was observed in $0.5 \mu \mathrm{g}$ and $1.5 \mu \mathrm{g}$ than in $2.5 \mu \mathrm{g}$. Therefore, the same experiment was repeated with $0.5 \mu \mathrm{g}, 1.0 \mu \mathrm{g}$, and $1.5 \mu \mathrm{g}$ of crude Ag. Since the values were almost similar for all three concentrations, the middle value, i.e., $1.0 \mu \mathrm{g}$, was selected as the best.

Using the optimized quantities of crude $\mathrm{Ag}(1.0 \mu \mathrm{g} / \mathrm{well})$, primary $\mathrm{Ab}$ concentration was optimized as described. BR was increased with the concentration of primary antibodies while secondary antibodies were decreased (Figure 3). According to the data obtained for ELISA absorbance values, $1: 1000$ and $1: 64000$ were selected as the best dilutions for serum (primary $\mathrm{Ab}$ ) and anti-species conjugate (secondary $\mathrm{Ab})$, respectively, where both $\mathrm{BR}$ and absorbance values were high.

In the pre-evaluation of specificity of the optimized ELISA method, high absorbance values were observed with 


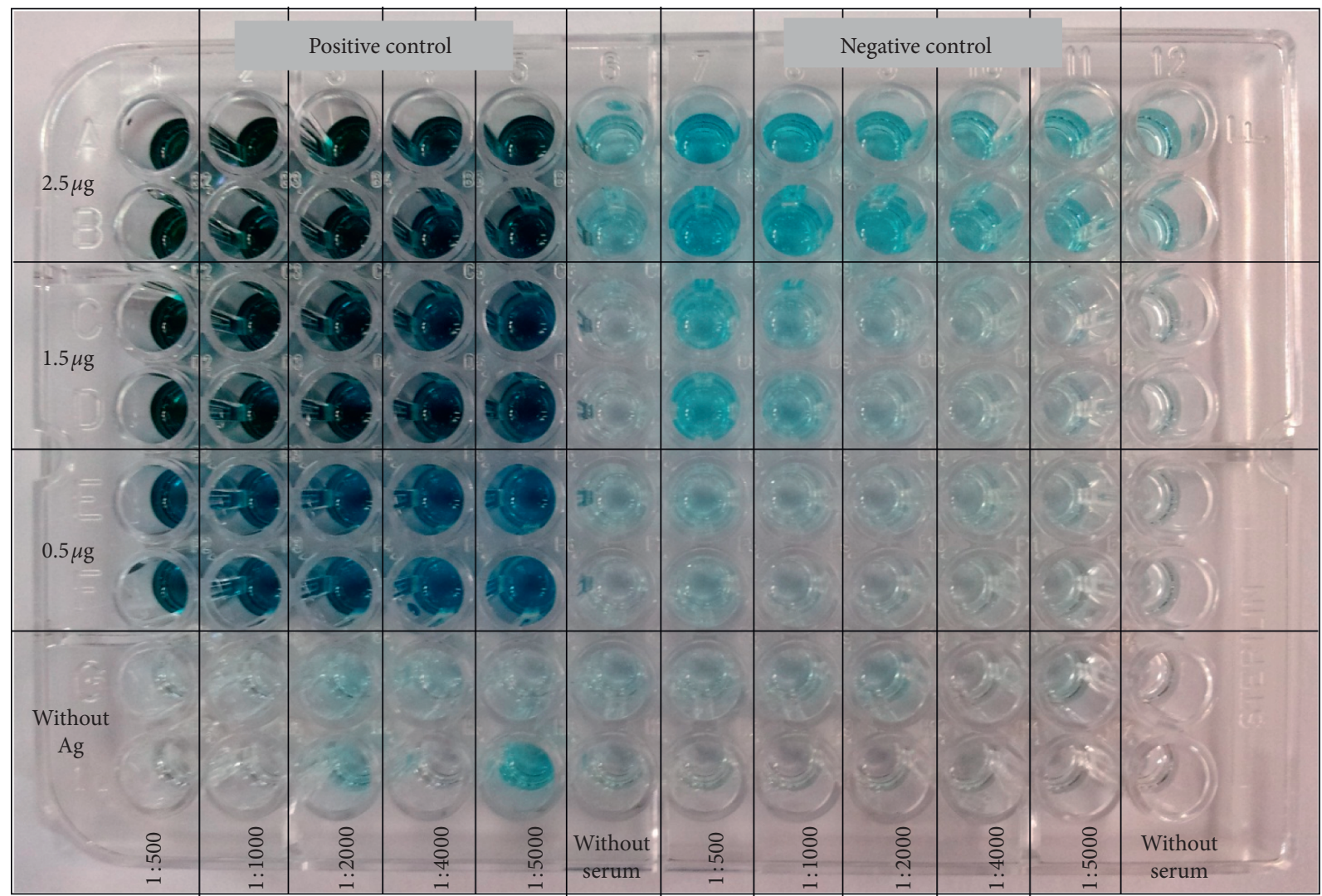

FIgURE 2: CBT of crude Ag concentration against serum after 30 minutes from adding TMB substrate solution. Ag was diluted rowwise, and positive and negative sera were diluted columnwise. Duplicates were done for each condition.

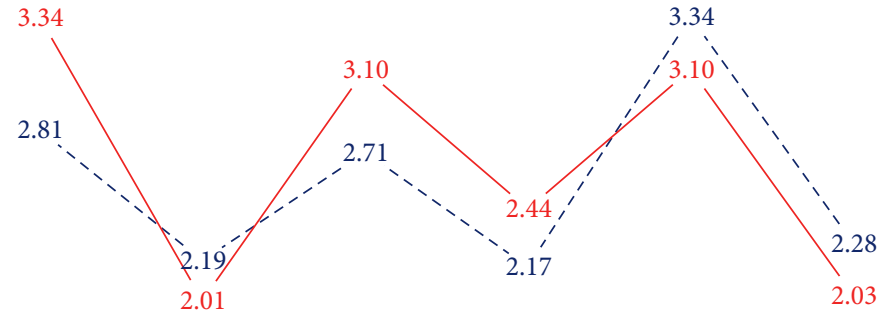

\begin{tabular}{|c|c|c|c|c|c|c|}
\hline Sample: & $\mathrm{P} 1$ & P2 & $\mathrm{P} 1$ & P2 & P1 & P2 \\
\hline$($ Primary Ab): & & & & & & \\
\hline
\end{tabular}

(Secondary $\mathrm{Ab})$ :

(a)

Figure 3: Continued. 


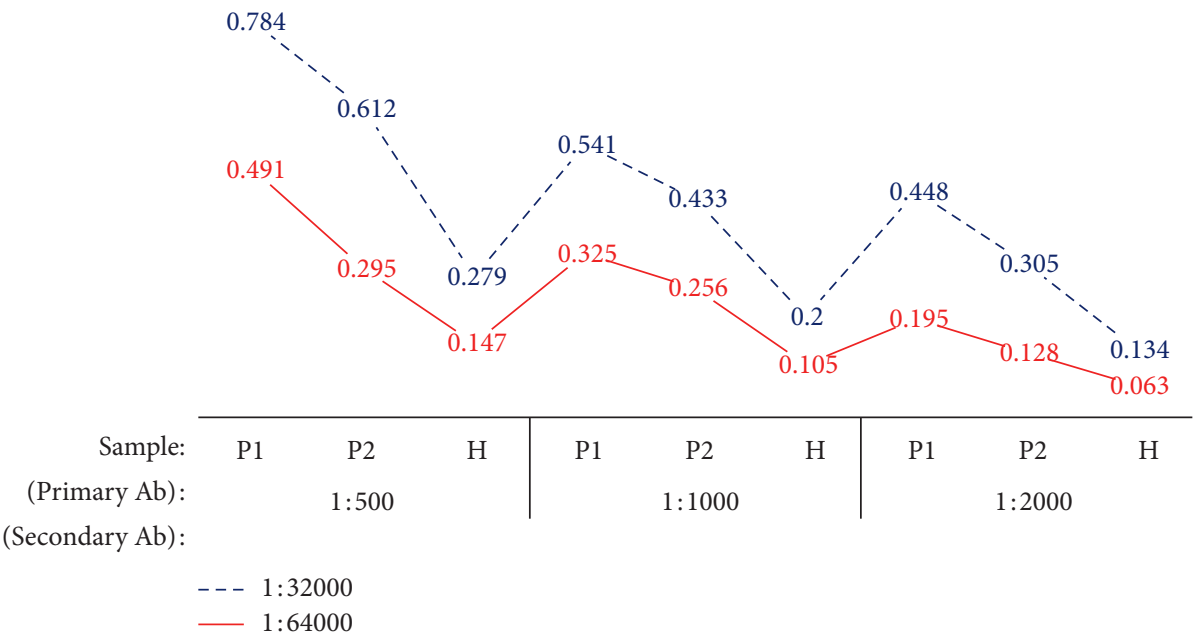

(b)

FIGURE 3: The variation of BR and ELISA absorbance values in different concentrations of primary and secondary Ab concentration (P1: high positive serum, P2: low positive serum, and H: healthy serum). (a) Variation of BR. (b) Variation of ELISA absorbance values.

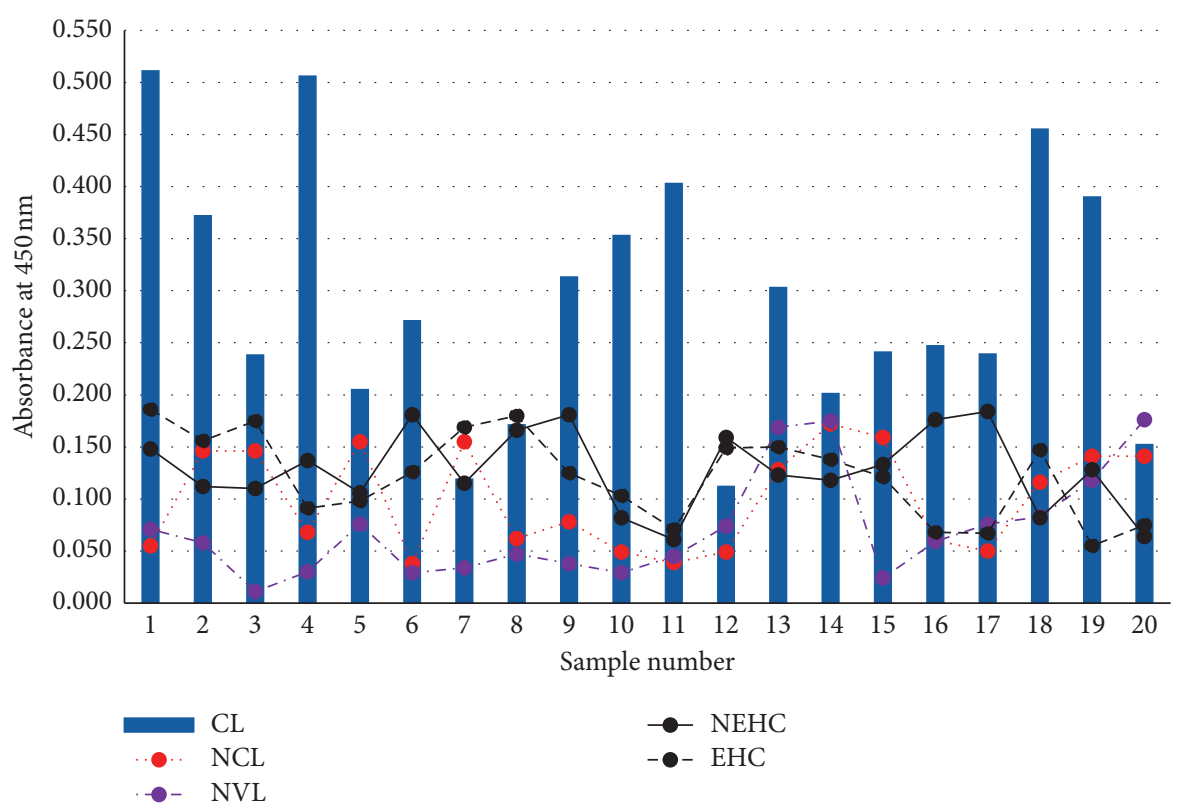

FIgURE 4: ELISA absorbance values of CL positives and other control samples. Twenty different samples from each group were used for comparison.

CL sera compared with control samples except 4 out of 20 CL sera which showed comparably lower absorbance values than other CL sera (Figure 4).

\section{Discussion}

In this study, we further optimized and examined an inhouse ELISA which is important to evaluate the humoral response in leishmaniasis in settings with $L$. donovani-induced CL.

Among different methods of ELISA, the indirect method was selected as it was user-friendly and most suitable for the requirement compared with other ELISA methods [37]. The indirect ELISA method was developed for diagnosis of leishmaniasis in disease-endemic areas in other countries $[21,38,39]$. Because of its wide usage in diagnosis, the same technique optimized using local parasite $\mathrm{Ag}$ was applied on local CL cases.

In CBT, concentrations of two reagents were altered columnwise and rowwise on the microwell plate (Figure 2) at the same time. The concentrations at the best point which had high assay sensitivity were selected as the optimized concentrations for the two reagents [37]. Using the CBT method, the optimal concentrations for crude Ag, patient sera, and 
anti-species conjugate were optimized as $1 \mu \mathrm{g}, 1: 1000$, and 1 : 64000 , respectively. The dilutions of human sera used for ELISA are usually varied from $1: 500$ to $1: 1000[21,22]$. Therefore, 1:1000 was reasonable, and it may have high amount of disease specific Abs than using higher dilutions at more than $1: 1000$. The dilution of a secondary Ab was mainly dependent on the initial concentration of the stock solution, sample source, and disease under examination.

$\mathrm{BR}$ was the ratio between respective absorbance values obtained for the positive control and the healthy control [37]. We were able to overcome the errors occurred due to nonspecific binding in healthy controls using BR when analyzing the ELISA results. High concentrations of secondary Ab may cause a reduction in the BR by increasing the nonspecific amplifications in healthy controls. P1, P2, and $\mathrm{H}$ were analyzed and compared for the BR, and absorbance values are depicted in Figure 3. At the lower dilutions of secondary $\mathrm{Ab}$, the $\mathrm{BR}$ of $\mathrm{P} 2$ was reduced due to increased nonspecific bindings. Also, in the higher dilutions of secondary $\mathrm{Ab}$, because of the reduction of absorbance values, the $\mathrm{BR}$ ratio was reduced. The comparison of both $\mathrm{BR}$ and absorbance values of ELISA together was important to select the optimum dilutions for primary and secondary Abs.

We successfully applied the optimized method for a study group which consisted of $n=20$ sera from each category of CL, NCL, NVL, NEHC, and EHC to analyze the binding specificity of the assay. Out of the study group, $n=16 / 20$ CL (80\%) patients gave remarkably high absorbance for ELISA than healthy control sera (Figure 4) which could be useful for discrimination of seropositive cases from healthy individuals.

\section{Conclusions}

In the current study, we described crude Ag-based indirect ELISA through altering the concentration of reagents and changing several conditions which would be applicable for other ELISA systems also. This assay may be useful for detection of anti-Leishmania IgG in CL caused by $L$. donovani. The assay may be evaluated using a larger number of samples, in multiple settings, and for different clinical scenarios in leishmaniasis. Further studies are underway.

Applications were submitted for patenting at the $\mathrm{Na}$ tional Intellectual Property Office of Sri Lanka (national patent $\mathrm{LK} / \mathrm{P} / 1 / 19697)$.

\section{Data Availability}

The data supporting the conclusions of this article are included within the article. Other data have not been made available as they were not part of the ethics application and due to patient confidentiality.

\section{Ethical Approval}

The study received the approval from the Ethics Review Committee, Faculty of Medicine, University of Colombo.

\section{Consent}

Informed written consent was obtained from all the individuals prior to recruitment.

\section{Disclosure}

The content is solely the responsibility of the authors and does not necessarily represent the official views of the mentioned funding sources.

\section{Conflicts of Interest}

The authors declare that they have no conflicts of interest.

\section{Authors' Contributions}

Y. S. conceptualized, financed, guided B. D., and critically revised the manuscript. B. D., P. S., and Y. S. designed the project. B. D. carried out the laboratory work, data analysis, and wrote the first draft of paper. S. W., H. G., T. P. A., M. N. P., and S. S contributed to data acquisition and technical work. P. S. and V. C. provided laboratory facilities and technical guidance and reviewed the manuscript. S. S. revised the manuscript critically for important intellectual content. All authors agreed on the final manuscript.

\section{Acknowledgments}

We acknowledge Nilusha Priyanthi, Yasasmi R Gange, Sashika Dayananda, Thisari S Rathnayake, and Nirosha Pathirana for the technical assistance and Nadira Karunaweera for administrative and logistical support in this study. This research was supported by the University of Colombo research grant $(\mathrm{AP} / 3 /$ 2/2014/RG/13).

\section{References}

[1] J. Alvar, I. D. Velez, C. Bern et al., "Leishmaniasis worldwide and global estimates of its incidence," PLoS One, vol. 7, no. 5, Article ID e35671, 2012.

[2] N. L. Sharma, V. K. Mahajan, and A. K. Negi, "Epidemiology of a new focus of localized cutaneous leishmaniasis in Himachal Pradesh," The Journal of Communicable Diseases, vol. 37, no. 4, pp. 275-279, 2005.

[3] A. M. Babiker, S. Ravagnan, A. Fusaro et al., "Concomitant infection with Leishmania donovani and L. major in single ulcers of cutaneous leishmaniasis patients from Sudan," Journal of Tropical Medicine, vol. 2014, Article ID 170859, 8 pages, 2014.

[4] A. Özbilgin, M. Harman, M. Karakuş et al., "Leishmaniasis in Turkey: visceral and cutaneous leishmaniasis caused by Leishmania donovani in Turkey," Acta Tropica, vol. 173, pp. 90-96, 2017.

[5] H. V. Y. D Siriwardana, M. A. B. Deepachandi, S. Ranasinghe, P. Soysa, and N. D. Karunaweera, "Evidence for seroprevalence in human localized cutaneous leishmaniasis caused by Leishmania donovani in Sri Lanka," BioMed Research International, vol. 2018, Article ID 9320367, 7 pages, 2018.

[6] Ministry of Health, Epidemiological Bulletin,Pan American Health Organization, Washington, DC, USA, 2015.

[7] N. D. Karunaweera, F. Pratlong, H. V. Y. D. Siriwardane, R. L. Ihalamulla, and J. P. Dedet, "Sri Lankan cutaneous leishmaniasis is caused by Leishmania donovani zymodeme mon-37," Transactions of the Royal Society of Tropical Medicine and Hygiene, vol. 97, no. 4, pp. 380-381, 2003. 
[8] H. V. Y. D. Siriwardana, H. A. Noyes, N. J. Beeching, M. L. Chance, N. D. Karunaweera, and P. A. Bates, "Leishmania donovaniand cutaneous leishmaniasis, Sri Lanka," Emerging Infectious Diseases, vol. 13, no. 3, pp. 476-478, 2007.

[9] L. Yatawara, S. Wickramasinghe, R. P. V. J. Rajapakse et al., "Morphological and molecular studies on Sri Lankan leishmania," Tropical Medicine and Health, vol. 36, no. 4, pp. 171-179, 2008.

[10] M. Z. Alam, C. Haralambous, K. Kuhls et al., "Te paraphyletic composition of Leishmania donovani zymodeme mon-37 revealed by multilocus microsatellite typing," Microbes and Infection, vol. 11, no. 6-7, pp. 707-715, 2009.

[11] H. V. Siriwardena, C. U. Udagedara, and N. D. Karunaweera, "Clinical features, risk factors and efficacy of cryotherapy in cutaneous leishmaniasis in Sri Lanka," Ceylon Medical Journal, vol. 48, no. 1, pp. 10-12, 2003.

[12] H. V. Y. D. Siriwardana, N. Thalagala, and N. D. Karunaweera, "Clinical and epidemiological studies on the cutaneous leishmaniasis caused by leishmania (leishmania) donovaniin Sri Lanka," Annals of Tropical Medicine \& Parasitology, vol. 104, no. 3, pp. 213-223, 2010.

[13] S. N. Semage, K. P. N. Pathirana, and S. B. Agampodi, "Cutaneous leishmaniasis in mullaitivu, Sri Lanka: a missing endemic district in the leishmaniasis surveillance system," International Journal of Infectious Diseases, vol. 25, pp. 53-55, 2014.

[14] H. V. Y. D. Siriwardana, U. Senarath, P. H. Chandrawansa, and N. D. Karunaweera, "Use of a clinical tool for screening and diagnosis of cutaneous leishmaniasis in Sri Lanka," Pathogens and Global Health, vol. 109, no. 4, pp. 174-183, 2015.

[15] N. Samaranayake, S. D. Fernando, N. F. Neththikumara et al., "Association of HLA class I and II genes with cutaneous leishmaniasis: a case control study from Sri Lanka and a systematic review," BMC Infectious Diseases, vol. 16, p. 292, 2016.

[16] H. V. Y. D. Siriwardana, G. Zhou, M. A. B. Deepachandi et al., "Trends in recently emerged Leishmania donovani induced cutaneous leishmaniasis, Sri Lanka, for first 13 years," BioMed Research International, vol. 2019, Article ID 4093603, 11 pages, 2019.

[17] U. Sharma and S. Singh, "Immunobiology of leishmaniasis," Indian Journal of Experimental Biology, vol. 47, no. 6, pp. 412-423, 2009.

[18] S. Saha, T. Mazumdar, K. Anam et al., "Leishmania promastigote membrane antigen-based enzyme-linked immunosorbent assay and immunoblotting for differential diagnosis of Indian post-kala-azar dermal leishmaniasis," Journal of Clinical Microbiology, vol. 43, no. 3, pp. 1269-1277, 2004.

[19] E. Y. D. Rosário, O. Genaro, J. C. França-Silva et al., "Evaluation of enzyme-linked immunosorbent assay using crude leishmania and recombinant antigens as a diagnostic marker for canine visceral leishmaniasis," Memórias Do Instituto Oswaldo Cruz, vol. 100, no. 2, pp. 197-203, 2005.

[20] S. Sundar, R. Maurya, R. K. Singh et al., "Rapid, noninvasive diagnosis of visceral leishmaniasis in India: comparison of two immunochromatographic strip tests for detection of anti-K39 antibody," Journal of Clinical Microbiology, vol. 44, no. 1, pp. 251-253, 2006.

[21] S. Saha, R. Goswami, N. Pramanik et al., "Easy test for visceral leishmaniasis and post-kala-azar dermal leishmaniasis," Emerging Infectious Diseases, vol. 17, no. 7, pp. 1304-1306, 2011.
[22] D. Mukhopadhyay, N. K. Das, S. De Sarkar et al., "Evaluation of serological markers to monitor the disease status of Indian post kala-azar dermal leishmaniasis," Transactions of the Royal Society of Tropical Medicine and Hygiene, vol. 106, no. 11, pp. 668-676, 2012.

[23] M. Svobodová, B. Alten, L. Zídková et al., "Cutaneous leishmaniasis caused by Leishmania infantum transmitted by Phlebotomus tobbi," International Journal for Parasitology, vol. 39, no. 2, pp. 251-256, 2009.

[24] N. Sharma, V. Mahajan, A. Negi, and G. Verma, "The rK39 immunochromatic dipstick testing: a study for K39 seroprevalence in dogs and human leishmaniasis patients for possible animal reservoir of cutaneous and visceral leishmaniasis in endemic focus of satluj river valley of Himachal Pradesh (India)," Indian Journal of Dermatology, Venereology and Leprology, vol. 75, no. 1, pp. 52-55, 2009.

[25] H. V. Y. D. Siriwardana, G. S. P. Ranasinghe, S. Weerasinghe, S. S. S. B. D. P. Soysa, and N. D. Karunaweera, "A study on the visceralization potential of cutaneous leishmaniasis in Sri Lanka," Annual Research Symposium, vol. 232, 2012.

[26] D. Rathnayake, R. R. Ranawake, G. Sirimanna, Y. Siriwardhane, N. Karunaweera, and R. De Silva, "Co-infection of mucosal leishmaniasis and extra pulmonary tuberculosis in a patient with inherent immune deficiency," International Journal of Dermatology, vol. 49, no. 5, pp. 549-551, 2010.

[27] H. V. Y. D Siriwardana, P. Karunanayake, L. Goonerathne, and N. D. Karunaweera, "Emergence of visceral leishmaniasis in Sri Lanka: a newly established health threat," Pathogens and Global Health, vol. 111, no. 6, pp. 317-326, 2017.

[28] P. Manokaran, S. Sivansuthan, G. J. Pratheepan, and R. Gajanthan, "Locally acquired visceral leishmaniasis- first reported case in northern province of Sri Lanka," International Journal of Medical Microbiology and Tropical Diseases, vol. 3, no. 3, pp. 133-134, 2017.

[29] H. V. Y. D. Siriwardana, M. A. B. Deepachandi, C. Gunasekara, W. Warnasooriya, and N. D. Karunaweera, "Leishmania donovani induced cutaneous leishmaniasis: an insight in to atypical clinical variants in Sri Lanka," Journal of Tropical Medicine, vol. 2019, Article ID 4538597, 11 pages, 2019.

[30] H. V. Y. D. Siriwardana, M. A. B. Deepachandi, S. D. S. Weliange et al., "First evidence for two independent and different leishmaniasis transmission foci in Sri Lanka: recent introduction or long-term existence?" Journal of Tropical Medicine, vol. 2019, Article ID 6475939, 11 pages, 2019.

[31] R. L. Ihalamulla, H. V. Y. D. Siriwardana, and N. D. Karunaweera, "Efficacy of RPMI 1640 and m 199 media in the isolation of leishmania from cutaneous lesions," Annals of Tropical Medicine \& Parasitology, vol. 102, no. 2, pp. 173-175, 2008.

[32] L. Lachaud, E. Chabbert, P. Dubessay, J. Reynes, J. Lamothe, and P. Bastien, "Comparison of various sample preparation methods for PCR diagnosis of visceral leishmaniasis using peripheral blood," Journal of Clinical Microbiology, vol. 39, no. 2, pp. 613-617, 2001.

[33] M. A. B. Deepachandi, S. Weerasinghe, P. Soysa, N. Karunaweera, and H. V. Y. D. Siriwardana, "A highly sensitive modified nested PCR to enhance case detection in leishmaniasis," BMC Infectious Diseases, vol. 19, no. 1, p. 623, 2019.

[34] F. Afrin, R. Rajesh, K. Anam, M. Gopinath, S. Pal, and N. Ali, "Characterization of Leishmania donovani antigens 
encapsulated in liposomes that induce protective immunity in $\mathrm{BALB} / \mathrm{c}$ mice," Infection and Immunity, vol. 70, no. 12, pp. 6697-6706, 2002.

[35] R. Barbosa-De-Deus, M. L. Dos Mares-Guia, A. Z. Nunes et al., "Leishmania major-like antigen for specific and sensitive serodiagnosis of human and canine visceral leishmaniasis," Clinical and Vaccine Immunology, vol. 9, no. 6, pp. 1361-1366, 2002.

[36] M. A. B. Deepachandi, S. Weerasinghe, T. P. Andrahennadi et al., "Quantification of soluble or insoluble fractions of Leishmania parasite proteins in microvolume applications: a simplification to standard lowry assay," International Journal of Analytical Chemistry, vol. 2020, Article ID 6129132, 8 pages, 2020.

[37] J. R. Crowther, The ELISA Guidebook, Springer, New York City, NY, USA, Second edition, 2009.

[38] K. M. Kurkjian, L. E. Vaz, R. Haque et al., "Application of an improved method for the recombinant k39 enzyme-linked immunosorbent assay to detect visceral leishmaniasis disease and infection in Bangladesh," Clinical Diagnostic Laboratory Immunology, vol. 12, no. 12, pp. 1410-1415, 2005.

[39] S. A. Al-Nahhas, "Serodiagnosis of cutaneous leishmaniasis in the syrian arab republic," Saudi Medical Journal, vol. 30, no. 3, pp. 382-386, 2009. 